\title{
TAKE BACK CONTROL OF YOUR TIME: DESIGN ACTIVITY FOR REFLECTING ON TIME AND TIMEKEEPING PRACTICES
}

\author{
Mert YILDIZ ${ }^{1}$, Sila UMULU², Aykut COSKUN ${ }^{1}$ and Bahar SENER ${ }^{2}$ \\ ${ }^{1}$ Koç University, Media and Visual Arts Department, Turkey \\ ${ }^{2}$ Middle East Technical University, Department of Industrial Design, Turkey
}

\begin{abstract}
This paper reports on the procedure and the results of a design activity carried out to reflect on time and timekeeping practices. The design activity involved a diary study followed by an idea generation workshop in the scope of a graduate level course with the participation of 12 graduate level industrial design students. The paper first explains the procedure of the design activity. Then, it presents the outcomes under two themes: i) timekeeping related insights: the students' reflections on their timekeeping practices and ideas on how to improve these practices to support wellbeing; ii) pedagogical insights: students' thoughts and instructors' reflections on the online diary-keeping and conducting the workshop remotely. The findings are believed to be beneficial for inspiring designers interested in designing interventions to support better time management practices; and for design educators interested in conducting remote diary study and/or workshop in distance education settings.
\end{abstract}

\section{Keywords: Product design, timekeeping, online diary, wellbeing}

\section{INTRODUCTION}

Time is precious and one of the most valuable resources we have. It is often perceived as a resource that should be utilized or managed. How we plan and spend our time has a direct impact on our wellbeing. Challenging the traditional definitions of time [1] (e.g., time as a measure for productivity), rethinking chronobiological knowledge [2], devising new representations of time (e.g., using cycles of moon to illustrate time [3], and redefining time as a social coordination tool [4] are required to better understand time as a concept that we inherently experience in our daily lives, and to support better time management practices. The impact of time management practices on wellbeing is prevalent in educational contexts. For university students, time-related demands such as high workload, time pressure, and the challenge of self-organized learning are associated with elevated stress levels [5], but better time management practices are known to decrease the perceived stress [6]. The pressure put by such demands on students' wellbeing has been recently increased by the COVID-19 pandemic, as it led to a 'forced' transition towards engaging in remote learning practices. Hence, understanding students' experiences with their time management is crucial to create possible interventions that would support their wellbeing during and post-pandemic times.

This paper reports on the procedure and the results of a design activity to reflect on time and timekeeping practices of university students with the following objectives: i) to explore how students' relationship with time has changed during the pandemic, and ii) to identify design opportunities and to ideate on potential routes for supporting 'good' time keeping practices that would bring positive impact on students' wellbeing. The design activity involved a diary study followed by an idea generation workshop in the scope of a graduate-level course at Middle East Technical University with the participation of 12 graduate level industrial design students, who all had industrial design background but completed their undergraduate studies at various universities. In the diary study, the students individually reflected on their time management and timekeeping habits during COVID-19 by filling in an online diary. They were asked to answer the following questions: 'Where does the work-life end, where does private life begin? How can we manage the boundaries between work time/non-work time in which the way we work, relax, and socialize have drastically changed due to the COVID-19 pandemic? Do we have control over our time? To which conflicts and tensions do our lack of control of our time lead?' Then, in the 
idea generation workshop, the students reflected on their perceptions of time and time management practices in groups and generated initial design proposals for supporting good practices.

Accordingly, the paper first explains the procedure of the design activity. Then, it presents the outcomes under two themes: i) timekeeping related insights: the students' reflections on their timekeeping practices and their ideas on how to improve these practices to support wellbeing; ii) pedagogical insights: students' thoughts and instructors' reflections on the methodological approach of conducting the diary study and the workshop remotely. The findings are believed to be beneficial for inspiring designers interested in designing interventions to support better time management practices; and for design educators interested in conducting remote diary study and/or workshop in distance education settings.

\section{DESIGN ACTIVITY}

The design activity was conducted in the context of a graduate-level course titled "Design for Sports, Health and Wellbeing". All students, who enrolled into the course, participated in design activity. The design activity was to enhance the students' understanding of wellbeing and to provide a starting point for the following phase of the course, in which students were asked to develop design concepts that improve wellbeing. The activity was carried out synchronously in two four-hour-sessions over Zoom. The sessions ran with a week apart. A general class discussion about time, time-management practices, and how COVID-19 impacted such practices, is carried out in the first session. Then, the students were introduced to the aim of the design activity. This was followed by a detailed presentation of the templates prepared for the students to fill in online. In the following six days, each student was asked to fill in their online diaries individually and asynchronously. In the second session, working in groups, the students generated proposals for supporting good timekeeping practices based on their input in the online diary. The following sections elaborate on both sessions.

\subsection{Diary-Keeping}

A six-day-long diary was devised to reflect on students' current understanding of time, and each day of the diary was specifically designed to challenge a unique concept of time (e.g., control of schedules, negotiation of time, right moments). The students were provided with an online Miro board space to individually fill in their diaries on the pre-prepared templates for six consecutive days. They filled in the templates by responding to set task(s). They were encouraged to take photos, videos, write notes, doodle etc. while reflecting on the certain aspects of 'time'. As a general approach, each day, the students were asked to record their timekeeping practices (any window throughout the day) and then to reflect on the recorded information (at the end of the day). For example, in day four, they were asked to reflect on their weekly schedules by visualizing the mismatches between "expected" and "real" durations of the calendar activities (e.g., meetings, shopping, online classes, etc.) that they allocated as time slots. The tasks in the templates were purposefully designed for quick reflections expected to take no more than 15 minutes a day. For example, in day one of the diary, one of the starting tasks was "name a favourite moment" followed up with a question asking, "what makes it a favourite moment?". Breakdown of the six days of the diary with their specific aims, tasks to be completed and a representative image from template(s) can be seen in Figure 1.

\subsection{Idea Generation Workshop}

In the idea generation workshop, students were divided into four groups of three. Groups were assigned to a new Miro board consisting of templates to facilitate the process (see Figure 2 for an example). To carry out the group activity, they were assigned to a Zoom Breakout Room (i.e., a feature in Zoom to help group of people to have a private chat), and regularly visited by the course instructors. The workshop was carried out in three steps: i) problem identification, ii) ideation, iii) discussion and reflection. In the first step, students were asked to share their diary entries with other group members. After all group members studied and briefly discussed each other's diaries, their collective reflections and problems identified in the diary study served as trigger to formulate a design problem. In the second step, they created a "How Might We" (HMW) question regarding this problem and generated multiple ideas. Then, they were asked to reduce the ideas into one and develop a user scenario of the selected idea. In the final step, all groups were invited to the Zoom main room to present their ideas. The groups also gave feedback to others' ideas and offered reflections on the workshop process. 


\section{INSIGHTS DERIVED FROM THE DESIGN ACTIVITY}

The design activity was analysed to reveal insights for solutions that support future timekeeping practices, as well as carrying out similar future activities in an educational setting. Quantitative analysis was not considered appropriate or necessary for the objectives at hand. The outcomes of the design activity (i.e., online diary entries and the design proposals) reviewed with an eye to find out whether there were any repeating themes across time checking practices, intersections, and ways of experiencing time. Diary activities also uncovered several issues pertaining to students' timekeeping practices which provided both students and instructors with insights into the topic. Course instructors reflected on their observations during the design activity and gathered them as insights on using online collaboration tools, online diary keeping and time management of the activity. Two themes were identified as a result: timekeeping related insights and pedagogical insights.

\subsection{Timekeeping-related Insights}

Timekeeping related insights were grouped under three sub-themes: fractured time checking practices, intersections of the schedules and activities, and ways of experiencing the progression of time.

\subsubsection{Fractured Time Checking Practices}

Student diaries revealed that current digital and/or app-based timekeeping technologies such as digital clocks and calendars, do not fully cover the richness of subjective perception of time. Some students expressed that checking time may sometimes cause stress, anxiety and even trigger negative feelings whilst interacting with such technologies. Besides, they mentioned that clocks/watches that represent time in numbers, minutes, and hours do not reflect on how and when to check time. The numerical representations of time often fail to capture the notion of "experiencing time as a whole" resulting in fractured time checking practices (e.g., looking at a clock but not perceiving anything meaningful or abandoning the digital calendars due to their overloaded content). Therefore, new ways of representing the concept of time are necessary to better grasp the notion of time checking, and to activate positive thoughts while checking time. In this respect, timekeeping technologies can make use of (personalized) "things" that are meaningful for that user. For example, several design proposals mentioned 'daily life activities' as new means of representing time (e.g., using coffee preparation process to visualize time). Accordingly, the students proposed that seeing upcoming events on a clock represented through new interactions (e.g., audial feedback, smell of something pleasurable) would be less stressful and enjoyable than just seeing the numbers on a clock.

\subsubsection{Intersections of the Schedules and Activities}

The main findings in this category can be summarized as: distinction between work and leisure time has been getting fuzzy; work time has been transforming into something that is extensively long and overwhelming; and leisure time is gradually disappearing that results in accumulation of negative emotions towards time. For example, while some students mentioned the problems, they face due to overwhelming work time (e.g., going into "depression sleep"), others raised the importance of equally valuing the leisure time with work time. Overall, students emphasized that current work time allocations are slot-based and that experiencing time through these slots (e.g., work time between 9:00-5:00 or meeting in 1:30 hours) causes negative emotions towards their perception of time. To address this problem, the students suggested rethinking timekeeping tools as smart agents. The calendar was given as an example to take an initiative and better communicate with its user, for example by indicating: "Hey John, you were checking out ... and I see an open slot, maybe you can book for this slot?". They indicated that such a solution would help them take back the control of time, which they have lost due to intersections between work and leisure related activities.

\subsubsection{Ways of Experiencing the Progression of Time}

As expressed in the students' diaries, timekeeping technologies (e.g., mobile phone apps that track task efficiencies based on timers) are not fully integrated into our lives. The students mentioned the necessity of experiencing the progression of time through new ways. Several students argued that technologies do not follow a natural cycle (e.g., waking up with the sunrise), for example they may need to wake up before the sunrise (to go to work or to attend a class). This makes them feel like they are no longer in control of their time. Natural cycles (e.g., growth of a plant) and daily life practices (e.g., going into a meeting) can be merged for better experiencing the progression of time. For example, several students 
mentioned using 'light' as a way of experiencing this progression, by emphasizing the concepts such as a clock that gets darker when the day and the night is approaching. Also, the progression of time can be represented through non-circular and non-repetitive clock designs. For example, "Tetris" can be an example for using it as a metaphor for illustrating the progression in activities. Lastly, students focused on "having an ability to be in the conversation" with timekeeping technologies as a way for better grasping the notion of progression.

\subsubsection{Students' Initial Design Proposals for Supporting Time Management Practices}

In the idea generation workshop, all four groups focused on different aspects of timekeeping related problems, and generated ideas responding to improved ways of spending time, meaningful representations for timekeeping achieved through the use technologies, and better ways of keeping control of the time. A brief description of the groups' proposals are as follows:

- Group 1: An activity-based calendar app that learns and adapts itself according to users' preferences. The app prioritizes the sequential activities and offers new ones based on the user's busyness level so that a more natural and uninterrupted schedule can be achieved.

- Group 2: A wellness assistant that aims to raise awareness for spending time more consciously. Whilst performing long tasks, the assistant tries to catch the user's attention by probing them with pop-up messages, and encourage them to give a break, then provides feedback on their use of time.

- Group 3: A smart watch coupled with a phone app to support better sleep times. The system learns the user's music taste and play songs in the right moments to signal the time to sleep/wake up.

- Group 4: A journal that contains several digital components to nudge its users to start a task. This was aimed to trigger the user's motivation to initiate a task with a reward system.

\subsection{Pedagogical Insights}

On the completion of the idea generation workshop, a questionnaire, consisting of several open-ended questions, were distributed to the students to gather their feedback regarding the design activity. They were specifically asked the usefulness of the diary-keeping; how they made use of their diary entries during the idea generation workshop; and whether they would like to advice any improvements. Their answers show that diary activities enabled them to better realize their time perception, the mismatches between what they might have planned and what they ended up doing during the day. Most students mentioned that diary-keeping was useful to identify problems relevant to idea generation workshop. However, some students mentioned their struggle to fill in the diary using an online platform, as it required carrying a device such as a laptop with them. Consequently, some students took their notes on paper sheets to be transferred into Miro later in the day. Finally, expressing themselves through photos and drawings found to be enjoyable and facilitated to convey students' thoughts and ideas regarding their conceptualization of time and time-related daily practices.

\subsubsection{Simultaneous Use of Zoom and Miro Programmes}

The idea generation session was conducted over Miro, partly synchronously (together with Zoom) and partly asynchronously (filling in diaries outside the class hours). However, during the class hours, Zoom and Miro were used simultaneously and collaboratively, which enabled group members to carry out their discussions on Zoom, while visualizing their ideas on Miro boards. Moreover, allocating breakout rooms for each group during the session helped students to keep their focus on their own task since they did not get distracted by other groups' conversations and shared their screen without interrupting the others. In summary, combining Zoom and Miro in the design activity was useful in terms of engaging students in idea generation tasks while maintaining the communication between different groups and instructors.

\subsubsection{Online vs Paper Based Diary-Keeping}

From the instructors' point of view, the main advantage of an online diary was to monitor students' progress on diary entries, which would otherwise have been difficult in paper-based ones. It was observed that some students preferred to make daily entries to their diaries, while some preferred to fill in every 2-3 days. This was due to some students' preference of taking notes on paper throughout the day and transfer them onto the Miro boards later in the day. Besides, the online diary gave the students a chance to express themselves in various ways using a combination of digital media including photos, clipart, and drawings (see Figure 3). Only diary-keeping through Miro supported the integration of sound, videos and gifs which otherwise can be difficult in paper-based versions. In the context of idea 
generation workshop, completing the diary entries online supported students to transfer their ideas and thoughts quickly (using the copy-paste feature), onto the group idea generation boards and templates without having a need to reproduce the same content. Miro allowed the content to be managed and presented through a single platform, which enabled students to use their time efficiently.

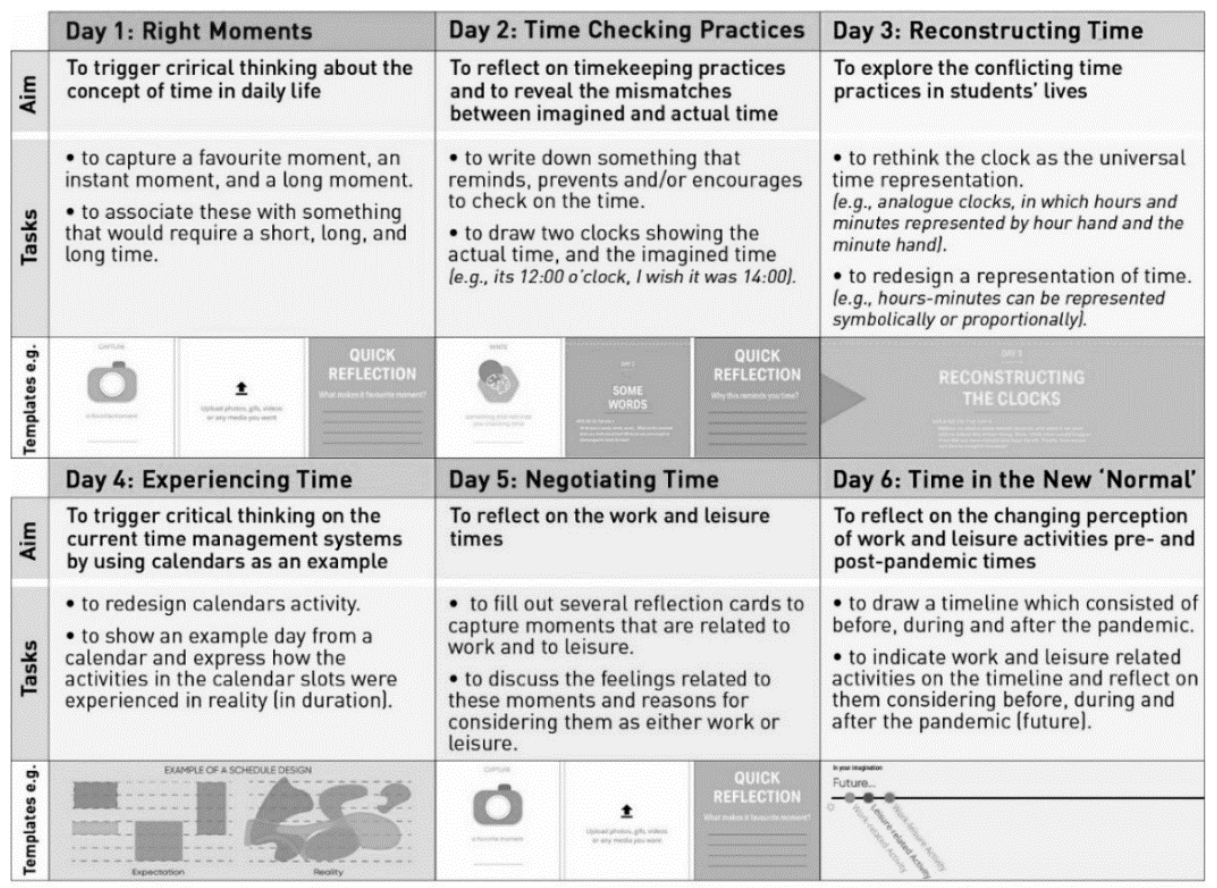

Figure 1. Aims, tasks to be completed, and example visuals from templates across the six diary days

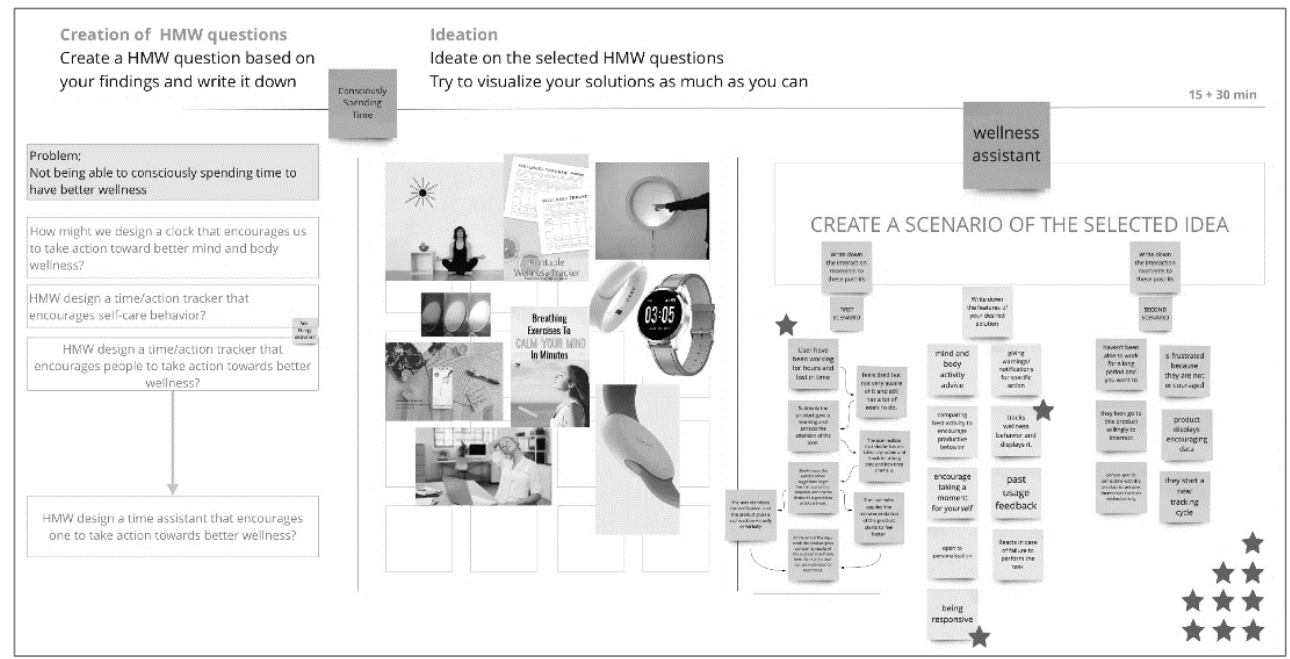

Figure 2. An example from a Miro board template filled in during the idea generation workshop

\subsubsection{Time Management of the Idea Generation Workshop}

The idea generation workshop was structured to provide flexibility to students and to balance online and offline activities. For example, the diary activities were designed to create an offline free time-space for students, and in-class activities (e.g., idea generation session) were designed to compile and have focused sessions. During in-class activities, apart from when it was necessary to brief the entire class, the students were provided with flexible time arrangement by means of dividing class hours with group activities, creating break-out rooms in Zoom, and having multiple breaks. This was thought to be necessary during an online education setting, where everybody stays in front of a computer screen for long hours even without noticing. The instructors observed that balancing online and offline activities and giving regular breaks in Zoom helped to keep the students' engagement high. This was confirmed 
by the questionnaire, administered to the students at the end of the semester to evaluate (anonymously) the different aspects of the course, including workshop. They were asked to rate (over 3-point Likert scale) whether they found the time and effort allocated for the workshop activities 'too much', 'just right' or 'too little'. Apart from one student, who commented that the time spent for diary-keeping in preparation for the workshop was 'too much', the remainder found it 'just right'.
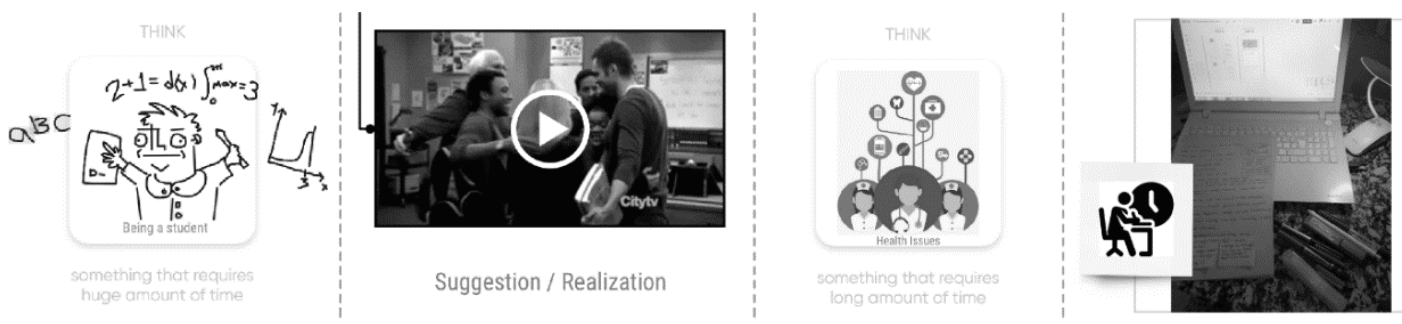

Figure 3. Examples of a drawing, a video, a stock image, and a captured image

\section{CONCLUSIONS}

This paper examined students' timekeeping and time management practices, and ways of supporting these practices with the help of a design activity (i.e., the combination of an online diary-keeping and a collaborative idea generation workshop) carried out with 12 graduate industrial design students. Insights gained to uncover: i) existing timekeeping tools fail to address fractured timekeeping practices as well as help understand the progressive nature of time, and ii) the intersections between work and leisure time blur the boundaries between the two and create a pressure on wellbeing through triggering negative emotions like anxiety. Furthermore, an activity organized in an educational setting integrating the dynamics of working individually/in a group, online/offline, and synchronously/ asynchronously together, helped to make useful observations for future set-ups. This diversity in task structures observed to be helpful for students to perform the tasks in their own pace (e.g., filling in the diaries outside the focused sessions) and stay focused on the task at hand while collaborating with others. Time and wellbeing are intertwined. This paper provided initial problem spaces that are related with timekeeping practices, we plan to further explore insights that are gained from graduate students. Besides, design activities showed the necessity of reflecting to the existing design course curricula for online education. We will utilize the insights related to this issue and will integrate in the future courses. In times of uncertainty, as the recent pandemic showed, the significance of better time-management practices for increased wellbeing becomes even more prominent. This paper was an effort to contribute to understand this relation from the perspective of university students and in an educational setting. The ideas generated during the design activity were also found be inspirational and diverse, helping to define ways to support timekeeping practices.

\section{ACKNOWLEDGEMENTS}

The authors would like to thank all graduate students who took part in the study.

\section{REFERENCES}

[1] Shih P. C., Bellotti V., Han K., and Carroll J. M. Unequal time for unequal value: Implications of differing motivations for participation in time banking. In Conference on Human Factors in Computing Systems, CHI'15, April 2015, pp 1075-1084.

[2] Janböcke S., Gawlitta A., Dörrenbächer J. and Hassenzahl M. Finding the inner clock: A chronobiology-based calendar. In Extended Abstracts of the 2020 CHI Conference on Human Factors in Computing Systems. CHI EA'20, April 2020, pp 1-7.

[3] Lee S. H., Kim S. B., Kim B. H., and Lee H. S. Lune: Representing lunar day by displayed lighting object. In Extended Abstracts of the 2019 CHI Conference on Human Factors in Computing Systems. CHI EA'19, May 2019, pp 1-4.

[4] Lindley S. E. Making Time. In Conference on Computer Supported Cooperative Work \& Social Computing, CSCW'15, February 2015, pp 1442-1452.

[5] Cotton S. J., Dollard M. F. and de Jonge J. Stress and student job design: satisfaction, well-being, and performance in university students. Int. Journal of Stress Management, 2002, 9(3), 147-162.

[6] Häfner A., Stock A., and Oberst V. Decreasing students' stress through time management training: An intervention study. European Journal of Psychology of Education, 2015, 30(1), 81-94. 